\title{
From an Inside-In towards an Outside-Out Urban Digital Twin: Business models and implementation challenges
}

\author{
Ruben D’Hauwers, (ruben.dhauwers@imec.be) ${ }^{1 *}$ \\ Nils Walravens, (nils.walravens@imec.be) ${ }^{1}$ \\ Pieter Ballon, (pieter.ballon@imec.be) ${ }^{1}$ \\ imec-SMIT-VUB, * corresponding author
}

KEY WORDS: Digital Twin. Business Model, Collaboration Model, Data Ecosystem, Data Governance, Smart City.

\begin{abstract}
:
Urban Digital Twins are a virtual representation of a city environment with bi-directional communication links. They require collaborations between different actors in the urban ecosystems in order to provide a complete picture of the situation in the city. In order to define the complex relationships between the different actors in the Urban Digital Twin ecosystem, the business model literature helps to answer questions on how value can be created, and how the value network can be controlled. In this paper, we identified four different types of business models for Urban Digital Twins based on whether they are used by the government or the ecosystem, and whether the government or the ecosystem controls the value network of the Urban Digital Twin. Interviews were held in five different existing Urban Digital Twins to identify which challenges the different existing digital twins have when implementing the Urban Digital Twins. The outcomes of the business model scenarios support the design of Urban Digital Twins 1) by identifying which decisions need to be made by cities when developing Urban Digital Twins and 2) by proposing cloud requirements for technology providers supporting cities, in the development of Urban Digital Twins.
\end{abstract}

\section{INTRODUCTION}

Cities have numerous challenges regarding climate change, mobility issues, a health crisis with the COVID-19 pandemic and dealing with the growing numbers of people living in the cities. Getting an overview of all the complexities in a city environment is crucial for ensuring that decisions can be made based on facts. Technology and data have some role to play in supporting or implementing policy (Hollands, 2008), but how that role should be filled remains unclear and is often the result of trial and error. The trend towards data-driven policymaking, which refers to policy decisions made based on objective empirical and evidence-based evaluation research about the context, need and efficacy of different policy programs rather than subjective intuition (Janssen and Helbig, 2018; Ruppert, Bernard and Kohlhammer, 2013) is raising in importance.

Getting a good overview of the city environment and the related data can support data driven policy significantly. A digital twin, a virtual representation of a physical entity with a bi-directional communication link (Tao et al, 2018), can become an important building block for getting this overview in the city in the form of an Urban Digital Twin. This is an urban decision support system which can support policymakers, public servants, urban planners, emergency responders and citizens in their decision making, and can help to interconnect various urban data sources and modelling algorithms in a way that can grow with the city and reflects its complexity (VLOCA, 2021). It could support use cases such as mapping traffic flows (Cityflows in Belgium ${ }^{1}$ ), support decision making concerning energy use and climate change (Helsinki in Finland ${ }^{2}$ ) and can help to visualize urban planning (Vienna in Austria ${ }^{3}$ ).

In an Urban Digital Twin, data needs to constantly be collected from different devices and stakeholders so that it can constantly capture changes and update the underlying data models of a digital twin. It encompasses 'urban data' such a contextual data, geographical data, traffic data, demographic data.... This data can be captured from Internet of Things solutions (e.g., sensors in public parking garages, passer-by sensors), privately owned data (E.g., transaction data of financial institutions...) or detailed data on the public domain (e.g., from satellite imaging).

Public players have access to open data sources and their own data, but the access to different Internet of Things and private data is limited. As the data is currently owned by different stakeholders, data silos which are not interconnected occur. To remove these data silos, data sharing in the data ecosystem may be required to support the cities to build digital twins. A potential support system for digital twins are data collaboratives, which are "cross-sector (and public-private) collaboration initiatives aimed at data collection, sharing, or processing for the purpose of addressing a societal challenge" (Susha et al., 2017).

The business model literature can shed a new light on the required collaborations to develop an Urban Digital Twin. Within the business model literature, a distinction can be made between authors that define a business model mostly on the level of the firm (Rappa, 2000; Osterwalder, 2004) while others define it at the network level (Weil and Vitale, 2001; Al-Debei and Avison, 2010; Timmers, 1998). On the network level of the firm, the main

\footnotetext{
${ }^{1}$ https://www.imeccityofthings.be/en/projecten/cityflows

${ }^{2}$ https://kartta.hel.fi/3d/atlas/\#/

${ }^{3}$ https://www.chnt.at/wp-content/uploads/Digital-geoTwin-Vienna.pdf

* Corresponding author
} 
questions to be solved relate to shifting firm boundaries, exploring the relationships that exist between actors in complex value networks and the roles they may play (Walravens and Ballon, 2013). As data ecosystems concern complex value networks, this research investigates the network level business models required for implementing Urban Digital Twin. It aims to provide insights on the following questions:

- $\quad$ What are the different types of business models of an Urban Digital Twin?

- What are the challenges digital twins have to implement the digital twin?

First, the approach of the paper will be discussed in Section 2. Based on business model literature, four scenarios of digital twins and their characteristics will be discussed in Section 3. These different challenges of digital twins will be discussed in Section 4. The conclusion will be covered in Section 5.

\section{APPROACH}

\subsection{Business model scenario building}

Different scenarios were made for identifying the different types of Urban Digital Twins. It is based on network-level business model parameters which explore the relationships that exist between actors in complex data ecosystems and the roles they may play. The overarching questions concern "Who controls the value network and the overall system design" and "Is substantial value being produced by this model" (Ballon, 2007). The chosen parameters are discussed in section 3.1 of this document. The scenarios are used to identify the characteristics of an Urban Digital Twin and to identify the challenges and the related requirements which are specific to the type of digital twin. The scenarios lead to four different types of Urban Digital Twins.

In each business model scenario, the characteristics of the Urban Digital Twin are described in order to clarify how the digital twin is set up. The characteristics are based on parameters defined by (Hartman et al., 2014), (Kampfer et al., 2019), (Gelhaar et al., 2021), (Ballon, 2007) and (D'Hauwers and Walravens, 2021). On the value parameters, the following characteristics are described:

- Purpose: The reason why the digital twin exists.

- User: Who is the user of the digital twin (a single firm or organization, the value chain, or entire ecosystem).

- Value proposition: Provision of (raw) data, information, and knowledge or a product or service.

- Added value activities: Generation, processing analysis, and visualization of data

The control parameters describe the following characteristics:

- Data type: Governmental open data, governmental closed data, commercial data or personal data.

- Data owner and data controller: Who owns the data and who controls the data.

- Control technical requirements: A single organization, the technology provider, or the ecosystem.

\subsection{Interviews with existing digital twins}

Six different existing Urban Digital Twins were interviewed in a semi-structured fashion. In order to select the digital twins to be interviewed, a shortlist with 25 digital twins in Europe, Asia, North America, and Australia was made. An initial analysis of the digital twins was made based on a desk research, where the purpose of the digital twin, stakeholders involved, data sources, and the business model complexity were assessed.

The actual selection of the Digital Twins was based on the geographical spread, the business model (data ecosystems were involved, which data types were used, and the number of stakeholders involved) and he availability of direct contacts to connect to the digital twin.

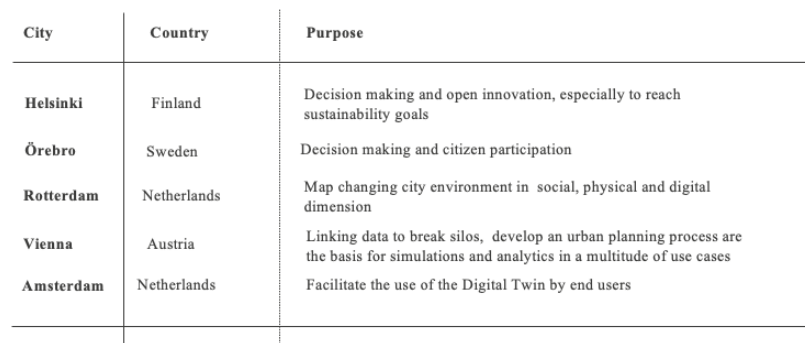

Table 1. Selection of Urban Digital Twins.

In order to develop the questionnaire for the interviews used for determining the challenges digital twins face in their business models, the data sharing business model framework was used (D'Hauwers and Walravens, 2021). It includes four main components:

- Value: How is value created and captured through financial models?

- Data governance: How is the quality, consistency, integrity, usability, security and availability of data ensured?

- Ecosystem trust: How is the trust in the ecosystem ensured?

- Data trust: How trust in what happens with the data ensured?

\section{DIFFERENT TYPES OF TWINS}

\subsection{Different types of digital twins}

The value proposition (D'Hauwers and Walravens, 2021) parameter, identifies by which actors the digital will be utilized. It could be used by the government, where the digital twin is used purely for policy making for the government in a closed model. In the other case, the digital twin is utilized by different actors in the data ecosystem, for e.g. citizen participation, for decision making in companies or citizens... in an open model. In figure 1 the y-axis moves from a closed model (solely used by the government) towards an open model (the data is open for anyone in the ecosystem.

The control parameters identify who controls data resources (Gelhaar et al., 2020). If control is central, the data resources are controlled by the government. In this case, the government is controlling the data resources and is also the main provider and controller of the data resources. The government is in this case also responsible for purchasing private data when required in bilateral agreements. If control is decentral, the data resources is controlled by the data ecosystem, in which case different actors in the data ecosystem are controlling the data resources and technology. Different actors serve in this case as data sources in an ecosystem. The different players in the data ecosystem can provide their conditions for sharing the data and control their data themselves. In Figure 1, the x-axis of control moves from a central control (the government controls their own data resources) towards a diffuse, decentral model of control 
(different stakeholders in the ecosystem provide their data and control their own data).

This results in four scenarios as shown in figure 1 and discussed below:

- Inside-In Urban Digital Twin

- Inside -Out Urban Digital Twin

- $\quad$ Outside - Out Urban Digital Twin

- $\quad$ Outside - In Urban Digital Twin

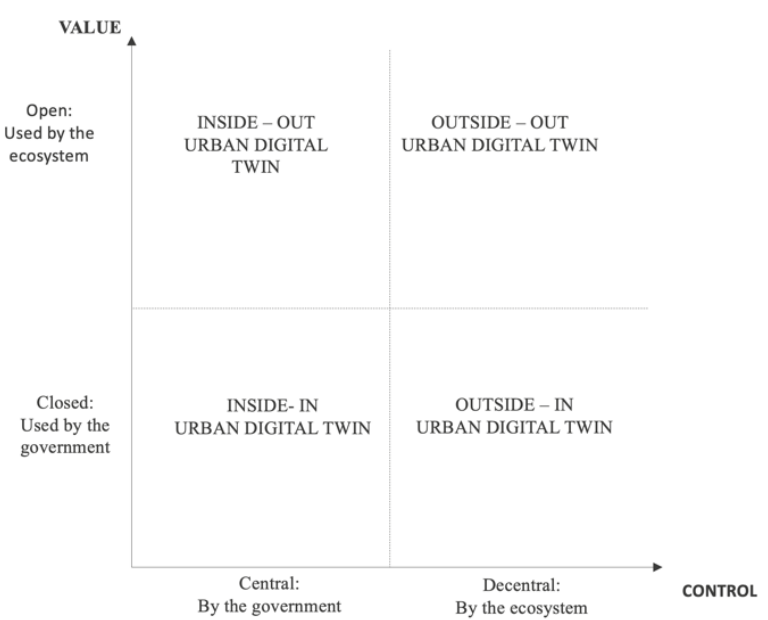

Figure 1. Urban Digital Twin business model scenarios.

\subsection{Inside-In Digital Twin}

The purpose of an Inside-In digital twin is to support the policymaking of the government, which is oriented purely on the internal governmental decision process. Some use cases in the Vienna digital twin are a good example of an Inside-In Digital Twin, as the purpose is to transfer data from reality to the virtual space in order to aid decision-makers and planners in their policymaking process.

Thus, these Urban Digital Twin use cases are not opened to the ecosystem. An Inside-In Urban Digital Twin can aid the decision process by visualizing data in the real world for policymakers, and thus can result in a better decision process when it is included in governmental planning for e.g., urban planning, climate change policies, and mobility policies. The Urban Digital Twin of Vienna (Lehner and Dorffner, 2020) is currently in the prototype phase. Therefore, one of the primary goals in Vienna is to break data silos within the government by linking data of different governmental sources. Second, the Urban Digital Twin of Vienna is first to be used in the in the urban planning process, and the goal is to aid in the development of new projects. It can showcase the effect of new infrastructure that is built and is used for simulations, such as for the development of the new SeesStadt Aspern urban development project and the North and North-West Train station area. Finally, the linking of the data and the urban planning process is the basis to enable analysis and simulations to make city-scale simulations such as climate, pollution, solar potential, flooding scenarios of the Danube...

The added value of the digital twin is to gather data from different governmental sources, process data in order to structure the data into a city data model and visualize the data in a real-world environment. In some cases, these Urban Digital Twins can also allow to make policy simulations.

In this scenario, the data source is mainly governmentally owned data, such as satellite pictures, Building Information Management data, point clouds, and other geodata such as, traffic data, and energy data. The model also aims to link databases from different governmental departments to the city model, such as data of the solar potential, climate data, flooding... The data is often generated and collected by the government and when needed are acquired on behalf of governmental organisations by private companies. The government is the owner of the data and thus also controls the data, and who can access the data.

The control of the technology used to develop the digital twin is controlled by the government. In most cases, they combine different open-source software into what is required for a digital twin. Most cities prefer not to purchase complete technological solutions from technology providers, as this limits the possibilities to adapt to changing requirements.

\subsection{Inside-Out Digital Twin}

The purpose of an Inside-Out Urban Digital Twin is to support the policymaking of the government by including the ecosystem in the decision process, and also to drive co-innovation, which is oriented to engaging the ecosystem to innovate with the data of the Urban Digital Twin. Thus, the digital twin is open to the ecosystem. A digital twin can aid the decision process by visualizing data in the real world for policymakers and for the ecosystem, and thus can result in a better decision process when it is included in governmental planning for e.g., urban planning, climate change policies, and mobility policies. An important goal of the digital twin of Örebro is to visualize data of the city to improve decision making and to include citizens in the decisionmaking of the city through citizen participation.

The Helsinki Energy and Climate Atlas, which is an open web service, built on a semantic digital twin model. It has four service modules: energy data, solar energy, heat demand, and geoenergy, can be used by companies, real estate developers, city planners, and building users. It is used by an energy advisory agency that advises people on renovations or on whether or not and where to install solar panels. Opening the city model data for the wider public can also support innovation in the ecosystem. In the Digital Twin of Helsinki, the city opens the data of the city model to anyone who can innovate using the data of the city. This was used in a challenge to how companies could use open data and $5 \mathrm{G}$ mobile networks, and how augmented reality could be useful in this challenge experiment.

The aim of 3D Amsterdam is to operate in a Plug \& Play Urban Digital Twin with a 3D platform to facilitate the use by end users. The objective is to disclose 3D data, visualize the data in 3D and to add open-source functionalities such as download and upload functions, drag and dropping of datasheets for making thematic 3D maps and the ability to make simulations. Thus, the role of the 3D Amsterdam is to provide an infrastructure for players in the ecosystem to use the Urban Digital Twin. Some use cases of 3D Amsterdam are to show the impact of a requested building permit on its surroundings helping the process of granting the permit, visualizations of thematic data and traffic flows or to give insight in the relationship between above ground urban developments and the underground infrastructure and soil.

In the case of the Urban Digital Twin of Vienna, a lot of governmental data is published as open data, as the City of 
Vienna has anchored the guiding principle "open by default" in its Data Excellence strategy. As Vienna is currently developing the Urban Digital Twin based on already existing data. Thus, if in the future the underlying data is open data, those parts of the digital twin will be open as well. This can for example be used by technical companies, urban planners, researchers, GIS consultants, interested citizens.... Vienna is working on a project similar to the building permit use case of 3D Amsterdam

The added value of the digital twin is to gather data from different governmental sources, process data in order to structure the data into a city data model and visualize the data in a real-world environment. In some cases, the digital twins also allow making simulations of a policy. The data can also be opened for the wider public to be used in innovative projects.

In this scenario, the data source is mainly governmentally owned data, such as satellite pictures, laser beams, Building Information Management data, point clouds, and other geodata such as telecom data, traffic data, and energy data. The data is often generated and collected by the government, and when needed purchased from private companies. The government is the owner of the data and thus also controls the data, and who can access the data. In this case, the government also needs to decide on which data can be open, and which data cannot be shared with the wider public. Reasons for not sharing the data can be because the data is sensitive, can pose negative effects on society when it comes into the wrong hands or simply because there are no use cases.

The technology used to develop the digital twin is controlled by the government. In most cases, they combine different opensource software into what is required for a digital twin. Most cities prefer not to purchase complete technological solutions from technology providers, as this limits the possibilities to adapt to changing requirements.

\subsection{Outside-Out Digital Twin}

The purpose of an Outside-Out Urban Digital Twin is to support the policymaking of the government by including the ecosystem in the decision process, and to drive co-innovation, which is oriented to engaging the ecosystem to innovate with the data of the Urban Digital Twin. Thus, the digital twin is open to the ecosystem. An Urban Digital Twin can aid the decision process by visualizing data in the real world for policymakers and for the ecosystem, and thus can result in a better decision process when it is included in governmental planning for e.g., urban planning, climate change policies, and mobility policies. Opening the city model data for the wider public can also support innovation in the ecosystem. The Rotterdam Digital Twin aims to integrate the changing city dimensions which moves from a social and physical construct towards a social, physical, and digital construct. The Urban Digital Twin aims to integrate a digital view of the reality of the city, serving as the base for smart solutions. Therefore, it aims to set up the digital infrastructure for a data ecosystem in Rotterdam to bring different actors together through the digital twin and the open urban platform by sharing data within the ecosystem. The digital twin is mainly used to engage the ecosystem with different use cases. It engages with the ecosystem, as it provides data insights for different players in the ecosystem to make their own decisions (e.g., information on building permits, support the firefighting department to indicate emergencies...), and to engage citizens in participation in urban construction processes (an AR application on construction sites, citizen participation by allowing to give feedback).
The added value of the digital twin is to gather data from different governmental sources, process data in order to structure the data into a city data model and visualize the data in a real-world environment. In some cases, the digital twins also allow making simulations of a policy. The data can also be opened for the wider public to be used in innovative projects.

In this scenario, the data source is from the data ecosystem, which combines data from governmental data sources, satellite pictures, laser beams, Building Information Management data, point clouds, and other geodata such as telecom data, traffic data, and energy data. The data is often generated and collected by the government, and when needed purchased from private companies. The data can also be obtained from the ecosystem, which could be done through a marketplace or through the engagement of different actors in the ecosystem. The ecosystem is the owner of the data and thus also controls the data, and who can access the data. This means that the actor can owe the data can decide which data will be opened for the digital twin, under which conditions. In this case, the different actors need to decide for themselves which data can be open, and which data cannot be shared with the wider public. Rotterdam aims to set up a data ecosystem called the Open Urban Platform. In this data ecosystem, the added value of the city is to connect different actors, to develop the platform, to own the platform, and to invest in the initial stages. After the first phase of setting up the platform, it might be transferred to the ecosystem itself. Once the platform is operational, the city and the data ecosystem will be able to act as a data provider, developer, user, and customer of the digital data ecosystem. Thus, the data sources of the Urban Digital Twin will be governmental data, as well as data from the data ecosystem gathered from the open urban platform. Also, the Örebro digital twin aims to set up a data ecosystem called Linked Örebro, which is still under development. The city of Vienna aims at an urban data space as well. Thus, ecosystem data will serve as an input for the digital twin. The usage of data of the ecosystem is guaranteed by mechanisms of data sovereignty.

The control of the technology to develop the Urban Digital Twin is controlled by the government but could be transferred to the ecosystem if this would be required in later stages. The government is often the initial innovator due to the investment requirements but could decide in a later phase to transfer the control to the ecosystem. In most cases, they combine different open-source software into what is required for a digital twin. Most cities prefer not to purchase complete technological solutions from technology providers, as this limits the possibilities to adapt to changing requirements.

\subsection{Outside-In Digital Twin}

The purpose of an Outside-In Urban Digital Twin is to support the policymaking of the government, which is oriented purely on the internal governmental decision process. Thus, the digital twin is not opened to the ecosystem. A digital twin can aid the decision process by visualizing data in the real world for policymakers, and thus can result in a better decision process when it is included in governmental planning for e.g. urban planning, climate change policies, and mobility policies. Some of the use cases of Örebro and Rotterdam could fit within the Outside-In Digital Twin. If the data is obtained from the data ecosystem, and the digital twin is used for governmental decision-making, it could be an example of an Inside-In digital twin. In Örebro this could be the case in the future, when the Linked Örebro data ecosystem will serve as a data input for the digital twin, for use cases related to urban planning. In the case of Rotterdam, this is the case for e.g. the flooding simulation use 
case when it is used for making governmental decisions based on the data.

In this scenario, the data source is from the data ecosystem, which combines data from governmental data sources, satellite pictures, laser beams, Building Information Management data, point clouds, and other geodata such as telecom data, traffic data, and energy data. The data is often generated and collected by the government, and when needed purchased from private companies. The data can also be obtained from the ecosystem, which could be done through a marketplace or through the engagement of different actors in the ecosystem. The ecosystem is the owner of the data and thus also controls the data, and who can access the data.

\subsection{Overview characteristics of the Digital Twin types}

Table 2 provides an overview of the characteristics of the digital twins discussed above.

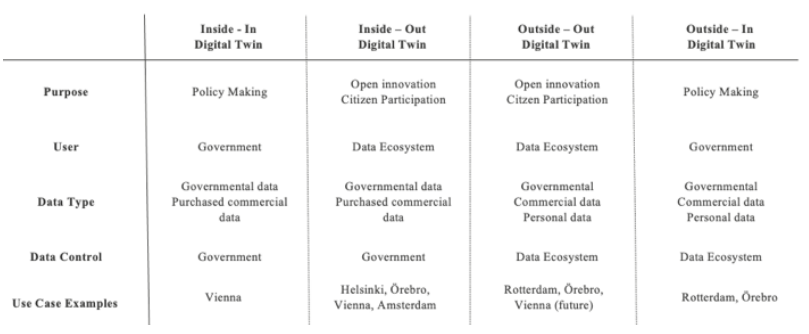

Table 2. Characteristics of the Urban Digital Twin Types.

\section{VALUE PROPOSITION AND REVENUE MODELS OF DIGITAL TWINS}

The value which can be created through the development of the digital twin can be internal or external. When the value is internal, the value is mainly captured by the government. This is the case in the Inside-In and Outside-Out Urban Digital Twin, and can concern improved governmental decisions, improve the data in the government, improve the communication, improve governmental services. In these cases, the Urban Digital Twins will always be paid by the government, as it can provide internal value. Thus, governmental departments will allocate budgets in order to reach their policy goals.

In the cases of the Inside-Out and Outside-Out Urban Digital Twin, the value can be internal (for the government) and external (to the ecosystem). Internal value can be a better service provision, reaching policy goals, improvement engagement and feedback of citizens. External value (for the ecosystem) can be for the citizens (to improve their decisions and get information and provide feedback) or by NGOs, companies in order to provide a better service offering to their customers. In these cases, in most of the Urban Digital Twin the government mostly still pays for the Urban Digital Twin, as it is used to reach their policy goals. In the future alternative revenue models could be identified when value is created for alternative stakeholders who would receive sufficient value to be willing to pay. In some cases, this could be for access to data, for a better service delivery or for access to use the Urban Digital Twin infrastructure (such as in the Plug and Play example from the City of Amsterdam).

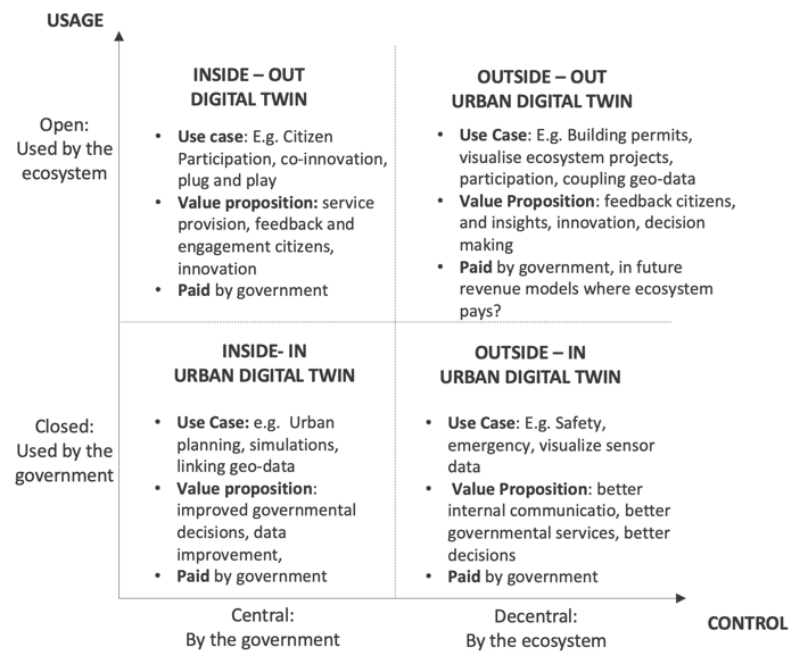

Figure 2. Value proposition Urban Digital Twins

\section{CHALLENGES DIFFERENT TYPES OF DIGITAL TWINS FACE}

In order to deliver on the value propositions discussed in section 4, different challenges need to be overcome in the implementation of the Urban Digital Twin. Based on the business model scenarios, different challenges can be identified in each type of Digital Twin, as shown in figure 3.

The basic challenge, which is the core for each business model scenario, is data governance. Having a structured information model and data governance is the core of the entire Urban Digital Twin development and is thus a challenge which is a basic requirement for the development of an Urban Digital Twin. When the data is opened to the ecosystem, decisions need to be made on which data can be shared with the ecosystem, and which data not. When the ecosystem is controlled by the ecosystem, and the data input is performed by the ecosystem, another challenge arose regarding ecosystem governance. The different challenges are discussed more in depth in the following sections 4.1 Data Governance, 4.2 Open Urban Digital Twin and 4.3 Ecosystem Governance. In section 5, some of these challenges are translated into cloud requirements which can be considered when developing technological solutions Urban Digital Twins.

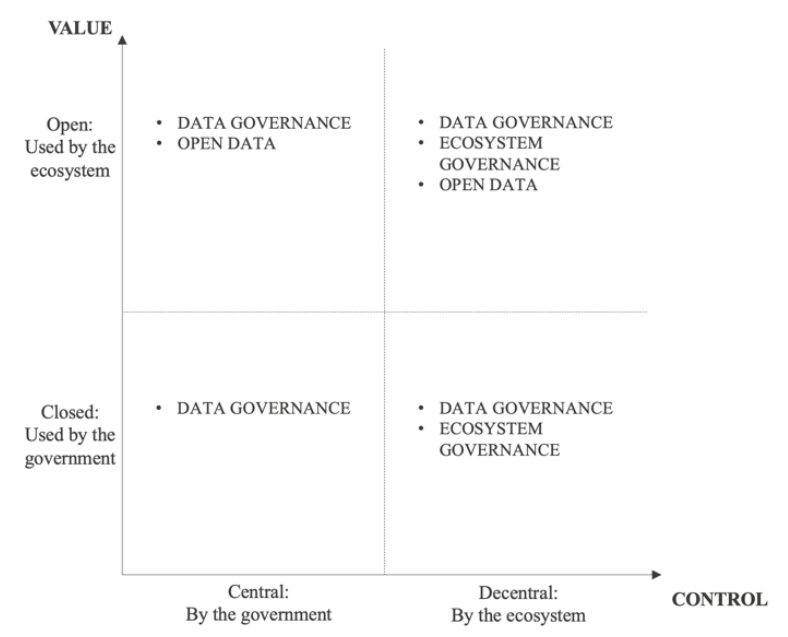

Figure 3. Urban Digital Twin implementation challenges. 


\subsection{Data Governance}

5.1.1 Organization: The collaboration between and within governmental departments is one of the major challenges when developing an Urban Digital Twin. The shift towards an Urban Digital Twin requires a cultural change. It requires not only to adopt new technology but also to adopt entirely new ways of working with completely different workflows compared to the past. New workflows are required in order to link the data and the database. It also requires collaborating with different departments who are not always willing to adapt their current way of working and to adopt the new standards. Additionally, new competencies are required which might currently not be present in the city.

5.1.2 Digital Twin Architecture and vendor lock-in: The data needs to be structured into a novel city information model, but there are no standards yet to implement this. CityGML is the only standard for city models, but it is not complete, and not all the standards of $3 \mathrm{D}$ classification are ready as there are no international standards yet. Additionally, there is no software yet which can cover the whole range of CityGML definitions. The different Urban Digital Twins are concerned about which cloud and other technological services to purchase and from which providers. They are very aware of the threats of vendor lock-in. Most Urban Digital Twins aim to not be dependent on the platforms of large companies and want to remain in control over the technological infrastructure and the used standards of the Digital Twin and open urban platform. Yet, when these components are developed in-house, this leads to a considerable amount of time and effort spent on developing their own infrastructure and reduced economies of scale.

5.1.3 Data availability and quality: A lot of the data that needs to feed into the architecture is spread in the whole organization in different departments. In some cities and use cases, the quality of the data is low and unstructured. It requires a lot of manual work and collaboration between the entire organization to structure and link the data. In some cases, the data does not exist or is not qualitative enough and needs to be purchased.

\subsection{Open Urban Digital Twin}

5.2.1 Classification of open data: In most of the cities, open by default is an important criterion. In order to open data, a classification of data is required which determines which data can be opened, and which data needs to remain closed. This depends, from city to city, on the confidentiality, correctness, and availability of the data. This depends also on the type of data, as governmental data is often seen as data which needs to be 'as open as it can be', whilst some data can be too sensitive to share (e.g. in the case of water piping data this can only be shared on a certain level of detail). In the case of commercial data, the sharing of the data depends on the conditions of the company. In the case of personal, privacy-sensitive data this needs to comply with the GDPR, and some cities even require setting up an ethical commission which needs to determine whether the data can be shared based on what will happen with the data and which data is required.
5.2.2 Identity Management: The identity management of the data determines who can access the data, and who can share the data. the city also needs to be able to control what happens with data on the level of the data source.

5.2.3 Open Data Standards: Often data can be opened by cities or companies but in some cases and cities the formats are unreadable for the entity who wants to utilise the data, or it might require licences to specific software which is not utilised by everyone and is therefore not useful. Therefore, there is a need for agreements on open data standards for opening Digital Twin data. Additionally, there is no (national or international) standard yet on visualization rules of $3 \mathrm{D}$ data. When offering $3 \mathrm{D}$ data models on a public 3D digital Twin, a city has to come up with their own rules to make the data visually understandable to prevent wrong interpretations.

\subsection{Ecosystem governance}

5.3.1 Governance Model: When the control over the data resources is allocated to the ecosystem, it requires a drastically changing role of the government. It needs to move from a more passive role towards taking an active role in the ecosystem and positioning the government and the data ecosystem actively. It requires a governance model for the ecosystem and a role definition for the government. Different activities will be required to facilitate the supply and demand of data in a marketplace, and additional services (such as data storage, geocoding...). Additionally, there is a role for marketplace governance who guards the balance between the commercial exploitation and the societally responsible behaviour of actors in the ecosystem. Furthermore, when the Urban Digital Twin is offered to the ecosystem as an infrastructure for the end users, questions arise on who gains value, who adds value and who owns the results of the outcome of the Urban Digital Twin. Therefore, when opening the digital twin to the ecosystem there can be questions on what the role of the city government, beneficiaries and contributors of the Urban Digital Twin are.

5.3.2 Trusted data ecosystem: A data ecosystem requires to ensure that the governance model is seen as a trusted data ecosystem. The data ecosystem needs to be willing to open the data, require clear data ownership rules which ensure control over the data resources and to set up conditions based on which the data can be shared. The trust between the data ecosystem is according to the interviewed Digital Twins one of the largest challenges to be set up in the Outside-Out and Outside-In Urban Digital Twin. In order to set this up, collaboration models need to be set up regarding the ownership of data, access to data, and open standards adoption by the ecosystem.

\section{URBAN DIGITAL TWIN CLOUD REQUIREMENTS}

Based on the challenges addressed in 5.1, 5.2 and 5.3, this section aims to translate these challenges into cloud business requirements. Some of the challenges the Urban Digital Twins face relate to the governance of the data, of the organisation within the cities and of the ecosystem around the Urban Digital Twin. These challenges cannot be addressed through Cloud Solutions, and require internal solutions performed by the city.

Other challenges can be seen as requirements for Cloud solutions for Urban Digital Twins. Thus, technology providers could offer technological cloud components which would enable the cities to 
focus on their internal challenges. In the centralized model where the government controls the data, the data will be gathered from different internal cloud servers which can be seen as internal data silos within the government. If it is gathered in a decentralized model where the data is controlled by the ecosystem, it will need to combine the access of data among different data silos in the ecosystem. Due to these silos, both in the centralised and decentralised models, cloud solutions can provide a solution for the cities. The main challenges that can be addressed through cloud solutions are shown in figure 4 .

\begin{tabular}{l|l|l} 
Challenge & Description & Cloud Requirement \\
\hline $\begin{array}{l}\text { Data Availability } \\
\text { and Quality }\end{array}$ & $\begin{array}{l}\text { (Internal and external) } \\
\text { data silos }\end{array}$ & Cloud Native Brokers \\
$\begin{array}{l}\text { Standardisation of } \\
\text { data and 3D } \\
\text { modelling }\end{array}$ & $\begin{array}{l}\text { Unreadable formats and licenses } \\
\text { which are not readable and no }\end{array}$ & Harmonisation of the data \\
$\begin{array}{l}\text { 3D standards } \\
\begin{array}{l}\text { Digital Twin } \\
\text { without risk of } \\
\text { vendor lock-in }\end{array}\end{array}$ & $\begin{array}{l}\text { Digital Twin infrastructure } \\
\text { which reduces the risk of } \\
\text { vendor-lock in }\end{array}$ & $\begin{array}{l}\text { Provide tools to develop } \\
\text { the Digital Twin internally } \\
\text { (standardisation, } \\
\text { procurement, exportation } \\
\text { of the data) }\end{array}$ \\
\hline
\end{tabular}

Figure 4. Urban Digital Twin cloud requirements.

6.1.1 Cloud Native Brokers In order to connect data sources, a digital component which connects data sources of cities (internally) and with data sources of the ecosystem (externally), which are called cloud native brokers. They can be seen as APIs which will configure and connect the different infrastructures. In the City of Things in Antwerp", a "context broker" has already enabled digital twins to connect different data sources, among others in a water use case. The context broker enables the digital twin to send and receive data in a structured way, which ensures readability.

6.1.2 Harmonization of data requires building standards of data (inter)nationally, to ensure all the data is structured in the same way. Microsoft and OASC have set up a Minimal Interoperable Mechanism (MIM) together as a Digital Twin Definition Language (DTDL) $)^{5}$ for models, not exclusively for Azure Digital Twins.

6.1.3 Digital Twin Infrastructure to avoid vendor lock-in. Technological tools will most probably not be sufficient to avoid vendor lock-in. Cities need tools to arm them in negotiations with technological providers. Different tools could be support to write a tender with conditions which avoid vendor lock-in. This way the cities can include these documents in negotiations with technology providers. This could include a clause which requests technology providers to ensure data export functionality, which ensures that governments can easily change towards another provider. Also, standardisation and harmonisation of the data (see above in other requirements) play a role in avoiding vendor lock-in.

\section{CONCLUSION}

When designing a digital twin, different decisions need to be made concerning who the user will be, what the purpose of the digital twin will be and how it can be governed. Decisions also

\footnotetext{
${ }^{4}$ https://www.imeccityofthings.be/nl/blog/schaalbare-architectuur-voorinternet-of-water-flanders
}

need to be made on whether the Urban Digital Twin use case needs to be opened and shared with stakeholders in the ecosystem, or whether it remains closed for policy making purposes only. Other decisions concern which data needs to be utilized, and who controls this data. The different types of Urban Digital Twins provide a framework for structuring the different design choices of use cases in Urban Digital Twins. The different types of Urban Digital Twins are the following:

- Inside-In Digital Twin

- Inside-Out Digital Twin

- Outside-Out Digital Twin

- Outside-In Digital Twin

The choice between these types of digital twins depends on the desired purpose, the data that needs to be included in the digital twin and the maturity of the digital twin. The four types of digital twins can co-exist, as it depends on the use case. Thus, in one city there can be the need for both an Inside-In Digital Twin for policy purposes, and an Outside-Out Digital Twin for ecosystem purposes.

First, for cities, these different types of Urban Digital Twin can guide the implementation process, as the chosen type has a major impact on the design of the Urban Digital Twin, and on the challenges that will need to be faced in the development of the Urban Digital Twin. The different challenges which the different types of digital twins face are shown in figure 5, as well as how it evolves when Urban Digital Twins move from one type of digital twin towards another. It can be seen as a maturity model where a Urban Digital Twin matures towards different types of Urban Digital Twins. The core challenge of any type of Urban Digital Twin is the data governance, and this is the foundation for developing the Urban Digital Twin. The data governance is the basis of any Urban Digital Twin. Once the data governance is set in place, a next challenge can be to open up the data to the entire ecosystem in the case of the Outside-Out and Inside-Out Urban Digital Twin, which requires a classification system of the data to determine which data can be shared with the ecosystem, and which needs to remain closed as well as an identity management solution. When the data supply and control of the data includes increasingly the ecosystem in the cases of the Outside-Out and the Outside-In Urban Digital Twin, another new challenge is the governance of the ecosystem, ensuring trusted data ecosystems and ecosystem governance models.

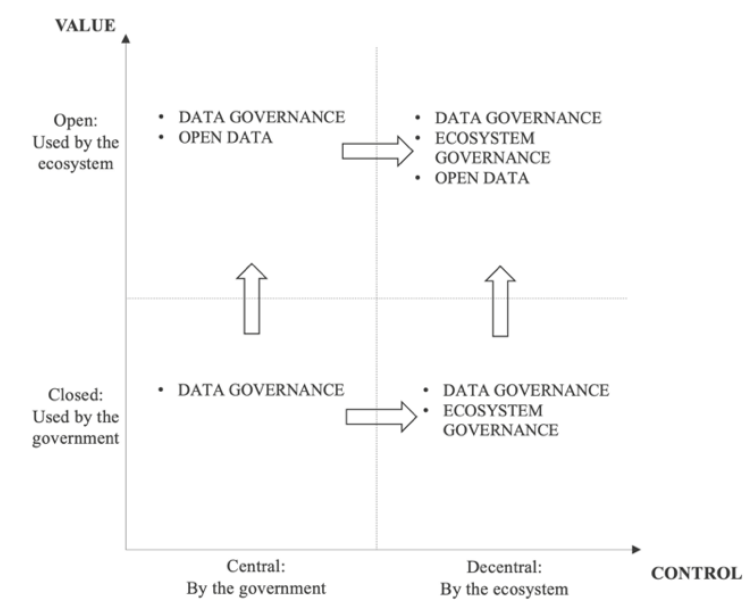

Figure 5. Urban Digital Twin challenge evolution.

\footnotetext{
${ }^{5}$ https://docs.microsoft.com/en-us/azure/digital-twins/concepts-models
} 
Second, for Cloud Solution Providers, the outcomes of the business model analysis shows different Cloud Business Requirements which can be developed by cloud providers and technology provides. The main components are the harmonization of data, cloud native brokers and the support in moving away from vendor lock-in challenges. This could be solved through technological solutions and through support in writing tenders for cities. Further research will investigate how this model can be utilized in the design of the Urban Digital Twins, and on how these challenges can be solved in the different scenarios.

\section{ACKNOWLEDGEMENTS}

The project DUET (https://www.digitalurbantwins.com) is funded by the European Commission. The interviews were held with representatives of the cities of Helsinki, Örebro, Rotterdam, Amsterdam and Vienna.

\section{REFERENCES}

Al-Debei, M., \& Avison, D. (2010). Developing a unified framework of the business model concept. European Journal of Information Systems, 19(3), 359-376.

Ballon, P. (2007). Business Modelling Revisited: The Configuration of Control and Value, Info - The Journal of Policy. Regulation and Strategy for Telecommunications, Information and Media, 9(5), 6-19.

D'Hauwers, R; Walravens, N. (2021). Do you trust me? Value and governance in data sharing business models, Springer, Proceedings from ICICT conference 2021

Gelhaar, Joshua \& Groß, Tobias \& Otto, Boris. (2021). A Taxonomy for Data Ecosystems. 10.24251/HICSS.2021.739.

Hartmann, Philipp \& Zaki, Mohamed \& Feldmann, Niels \& Neely, Andy. (2016). Capturing value from big data - a taxonomy of data-driven business models used by start-up firms. International Journal of Operations \& Production Management. 36. 1382 - 1406. 10.1108/IJOPM-02-2014-0098.

Hollands, R.. (2008). Will the Real Smart City Please Stand Up? City, 12 (3), pp. 303-320.

Janssen, M., \& Helbig, N. (2018). Innovating and changing the policy-cycle: Policymakers be prepared! Government Information Quarterly, 35(4), S99-S105.

Kampker, A. \& Stich, Volker \& Jussen, Philipp \& Moser, B. \& Kuntz, Jan. (2019). Business Models for Industrial Smart Services - The Example of a Digital Twin for a Product-ServiceSystem for Potato Harvesting.

Lehner, Hubert \& Dorffner, Lionel. (2020). Digital geoTwin Vienna: Towards a Digital Twin City as Geodata Hub. PFG Journal of Photogrammetry Remote Sensing and Geoinformation Science. 88. 10.1007/s41064-020-00101-4.

Osterwalder, A. (2004). The business model ontology: a proposition in design science approach. HEC Lausanne.
Rappa,
M. (2000).
Retrieved
from

Ruppert, T., Bernard, J., \& Kohlhammer, J. (2013). Bridging knowledge gaps in policy analysis with information visualization. Electronic Government and Electronic Participation-Joint Proceedings of Ongoing Research of IFIP EGOV and IFIP ePart 2022.

Tao, F., J. Cheng, Q. Qi, M. Zhang, H. Zhang, and F. Sui. (2018). Digital twin-driven product design, manufacturing and service with big data. The International Journal of Advanced Manufacturing Technology, 94(9-12), 3563-3576.

Timmers, P. (1998). Business models for Electronic Markets. Journal of Electronic Markets, 8, 3-8.

VLOCA. (2021). Retrieved on https://vlocakennishub.vlaanderen.be/vloca-

kennishub/Open_urban_digital_twins\#References)/ on the $2^{\text {nd }}$ of April 2021

Weil, P., \& Vitale, M. (2001). Place to Space: Migrating to eBusiness Models. Harvard Business School Press, 1. 